\title{
Using three-dimensional digital models to establish alveolar morphotype
}

\author{
M. Konvalinkova1, 2, W. Urbanova ${ }^{1}$, K. Langova ${ }^{3}$, M. Kotova ${ }^{1,2}$ \\ ${ }^{1}$ Department of Orthodontics and Cleft Defects, Third Faculty of Medicine Charles University and University Hospital \\ Kralovske Vinohrady, Prague, Czech Republic \\ 2Department of Orthodontics, Faculty of Medicine and Dentistry, Palacky University, Olomouc, Czech Republic \\ ${ }^{3}$ Department of Medical Biophysics, Faculty of Medicine and Dentistry, Palacky University, Olomouc, Czech Republic
}

[Received: 13 December 2017; Accepted: 29 January 2018]

\begin{abstract}
Background: The aim of the study was to propose a classification of alveolar morphotype and assess a relationship between extraction/non-extraction orthodontic treatment and changes to the alveolar process.

Materials and methods: Seventy-five subjects (mean age $=23.2, S D=5.1$ ) were selected. Areas of the sections of the alveolar process (ASAP) at three different levels $(0,2$, and $4 \mathrm{~mm})$ were measured on pre- and post-treatment three-dimensional digital models. Method reliability was analysed using Dahlberg's formula, intraclass correlation coefficient, and paired t-tests.

Results: The mean ASAP was smallest at level 0 and largest at level 4 . Pre-treatment ASAP $<773 \mathrm{~mm}^{2},<863.9 \mathrm{~mm}^{2}$, and $<881.1 \mathrm{~mm}^{2}$ at levels 0,2 , and $4 \mathrm{~mm}$, respectively, should be described as a "thin" alveolar morphotype. Regression models showed that pre-treatment ASAP was a predictor of the change of the alveolus during treatment only at level 2.

Conclusions: Patients for whom pre-treatment ASAP is $<773 \mathrm{~mm}^{2},<863.9 \mathrm{~mm}^{2}$, and $<881.1 \mathrm{~mm}^{2}$ at levels 0,2 , and $4 \mathrm{~mm}$, respectively, should be described as having a "thin" alveolar morphotype. In these patients, extraction treatment, associated with a decrease in the alveolus area, should be exercised with caution. (Folia Morphol 2018; 77, 3: 536-542)
\end{abstract}

Key words: alveolar process, digital model, scanning

\section{INTRODUCTION}

Initial orthodontic/orthognathic documentation includes intraoral and extraoral photographs, radiographic images, and plaster models of the dentition. Until recently, the documentation was mainly "analog." Proper storage and retrieval of information from the documentation in such a format is economically and spatially challenging. Digital technology is currently becoming an integral part of orthodontic/ /orthognathic diagnosis and treatment. The photographs and radiographs are often used in digital form, and plaster models are being quickly replaced by digital ones. In addition, three-dimensional (3D) radiography is indispensable in many patients. The digital revolution in orthodontics opens up new possibilities, particularly during treatment planning and quality control. For example, preparation of a diagnostic set-up, often a deciding factor during treatment plan selection, is quick and relatively easy when virtual models of dentition are used. The value of digital 3D documentation in neuro/craniofacial problems has been widely accepted [7, 10, 11, 23]. Another advantage of digital documentation is the facilitation of scientific research $[6,13,14]$. 
A recent population-based "Study of Health in Pomerania" [16] demonstrated that craniofacial morphology, specifically the cranial width and facial index, was a risk factor for periodontal problems gingival recession (GR) and clinical attachment loss (CAL). An 11-year follow-up of the sample comprising 556 healthy subjects from Pomerania, Germany, at a mean age of about 56 years at final observation, showed that the higher the facial index (i.e. ratio of facial height to facial width), the more pronounced were GR and CAL. In other words, subjects with the long narrow faces had about $0.3 \mathrm{~mm}$ more $G R$ and $0.5 \mathrm{~mm}$ more CAL compared to subjects with broad square faces.

The facial type is associated with morphology of the alveolus - subjects with long faces have a slender alveolus and thinner cortical bone compared to those with short faces who have a thick alveolar process with a dense cortical bone. Differences also concern facial growth - in a short facial type the maxilla grows more horizontally, whereas in a long facial type - vertical growth prevails over horizontal $[1,15,18]$.

The shape of the alveolus influences the range of possible teeth movements during orthodontic treatment because the cortical bone in the alveolar process creates a boundary beyond which teeth should not be moved. If the roots are forced outside of the cortical bone, bone dehiscence can develop [2]. The dehiscence can, in turn, lead directly to GR, especially when accompanied by the inflammatory process [21]. Measurement of the shape of the alveolus prior to treatment could help prevent the development of GR. Theoretically, tooth movement in subjects with a "thin" alveolar process should be less in comparison to those with a thick alveolus. Digital models made before treatment seem suitable for assessing alveolar morphology - they are relatively easy to obtain and, in contrast to radiographs, do not involve the patient being exposed to ionising radiation. Surface and volumetric analysis of models is also possible with numerous software packages. However, no clear description of what should be regarded as "thin" and "thick" alveoli, based on digital model measurements, exists in the current literature. Furthermore, it is important to establish how extraction in orthodontic treatment affects the morphology of the alveolus in comparison to non-extraction. Therefore, this study aims to: (a) propose a classification for the morphology of the alveolar process (alveolar morphotype), and (b) to assess the relationship between orthodontic treatment alternatives (extraction vs. non-extraction) and changes in the alveolus during treatment.

\section{MATERIALS AND METHODS}

The patient archives at the Department of Orthodontics and Cleft Anomalies, $3^{\text {rd }}$ Medical Faculty, Charles University, University Hospital "Královské Vinohrady", Prague, Czech Republic, were searched to identify subjects fulfilling the following inclusion criteria:

1. No systemic disease or congenital malformations;

2. Angle Class II malocclusion with normal (Class I) skeletal morphology before orthodontic treatment;

3. Flat ( $<1.5 \mathrm{~mm}$ deep) curve of Spee;

4. Permanent dentition in fully established occlusion;

5. No periodontal problems;

6. Orthodontic treatment with fixed appliances carried out after pubertal growth spurt;

7. Undamaged, good quality plaster models made before and after orthodontic treatment.

Seventy-five subjects ( 20 males, 55 females) were selected with a mean age of $23.2(S D=5.1$, ranging from 17.6 to 40.2 ) before orthodontic treatment (T1). Non-extraction treatment was performed in 39 subjects (15 subjects had protrusion of maxillary central incisors, i.e. were diagnosed with Angle Class II Division 1 malocclusion, and 24 subjects showed retrusion of maxillary central incisors, i.e. were diagnosed with Angle Class II Division 2 malocclusion). Extraction treatment (removal of maxillary first premolars) was carried out on 36 subjects (20 subjects had protrusion of maxillary central incisors, i.e. were diagnosed with Angle Class II Division 1 malocclusion; 16 subjects had retrusion of maxillary central incisors, i.e. were diagnosed with Angle Class II Division 2 malocclusion). Orthodontic treatment lasted for $1.7 \pm 0.3$ years (ranging from 1.1 to 2.4). Post-treatment (T2) documentation was carried out at a mean age of $24.9 \pm 5.0$ (ranging from 19.1 to 41.4 ).

One hundred and fifty plaster models (75 made at T1 and 75 at T2) were scanned with a D700 table top surface scanner (3Shape, Copenhagen, Denmark). The digital images were saved in open data format ( ${ }^{*}$ STL) and analysed using the 3Shape Ortho Analyser programme (3Shape, Copenhagen, Denmark).

The sagittal and occlusal planes were identified on the $\mathrm{T} 1$ and $\mathrm{T} 2$ digital models to orient the model in a 3D environment. Additionally, a virtual model base was formed before further analysis. The pre- 


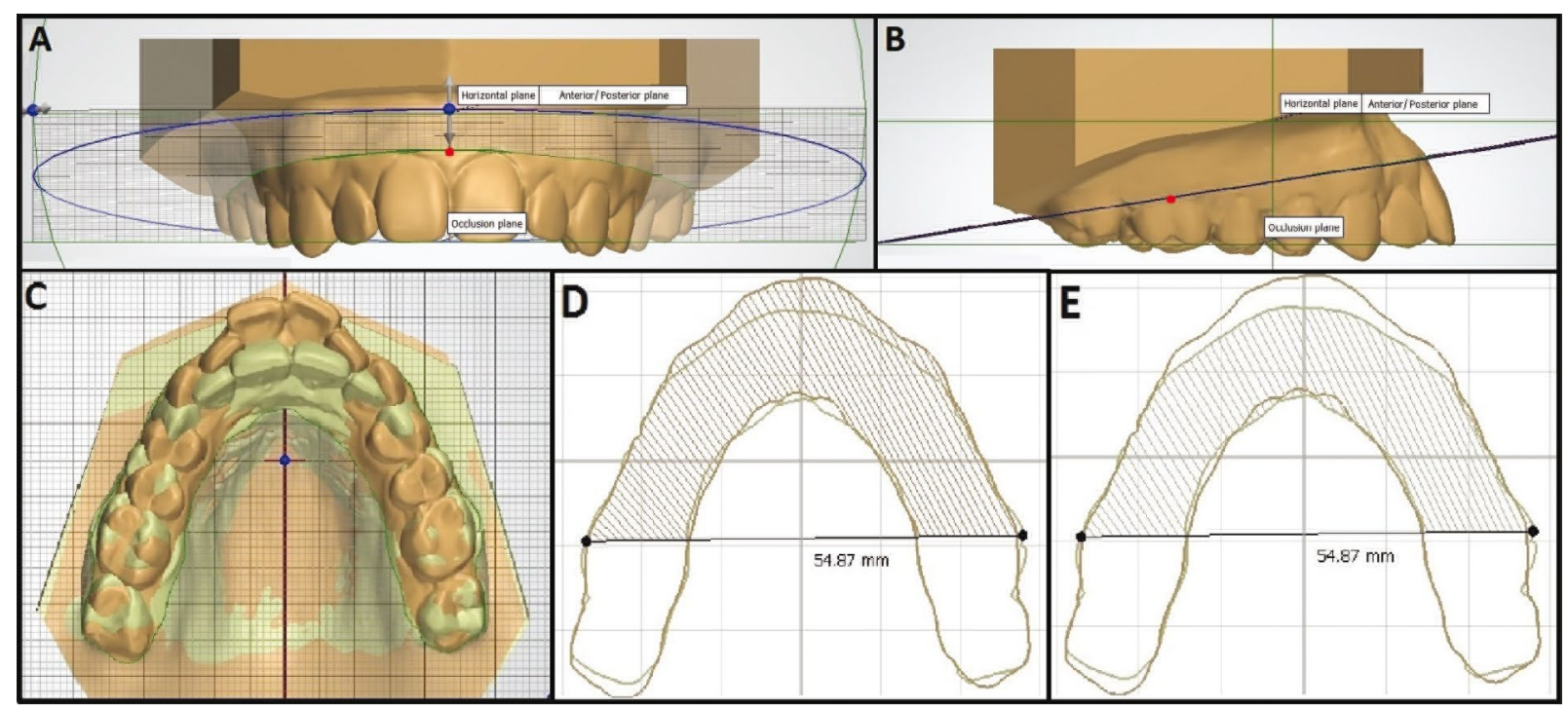

Figure 1. Definition of reference plane, three-dimensional superimposition of the images, delimitation of the measured area. A. Determining the reference level for transversal slices in the deepest point on the cementoenamel junction of the first molars and central incisors $=$ level 0 — frontal view; B. The reference level for transversal slices — lateral view; C. Superimposition of models before (brown colour) and after treatment (green colour) - occlusal view; D, E. Transversal slice at 0 level; the hatching represents the measured area on the model before treatment (D) and after treatment (E). The black line connects midpoints of the buccal side of the occlusal area of the right and left first molars $=$ the distal edge for measuring the area content of a given slice.

treatment (T1) reference level for horizontal slices was a plane determined by a point in the middle of the line connecting the deepest points on the cementoenamel junction of the central incisors (Fig. 1A), and the deepest points on the cementoenamel junction of the first molars bilaterally (Fig. 1B) (hereafter referred to as tooth cervical level 0) [12]. The two additional virtual slices were made parallel to this plane but at a distance of $2 \mathrm{~mm}$ (cervical level 2 ) and $4 \mathrm{~mm}$ (cervical level 4) apically from cervical level 0.

Scans of the models made before and after treatment were superimposed (Fig. 1C) with the help of individually determined orientation points. Rugae palatinae and palate relief were used as superimposition sites as they had been previously shown to be suitable for this purpose [3]. The areas defined by lines contouring the vestibular and palatal sides of the horizontal slices through the alveolar process at levels 0,2 , and $4 \mathrm{~mm}$ before treatment (Fig. 1D) and after treatment (Fig. 1E) were measured on the scans of the superimposed models. The distal border for measuring the area of the alveolar process on a given slice was a straight line connecting the midpoints on the buccal side of the occlusal surface of the right and left first molars identified on the T1 model. After superimposition, the original (T1) distal border reference line was transferred to the post-treatment (T2) digital model. Therefore, the movement of the first permanent molars during treatment did not influence the measurement area of the alveolar process (Fig. 1D, E). All models were scanned and measured by one investigator (first author). All measurements were performed with $1 / 100 \mathrm{~mm}$ precision.

\section{Reliability of the method and statistical analysis}

Twenty randomly selected models were once re-scanned, once re-superimposed, and then once remeasured by the same investigator (first author) after $>2$ weeks. Measurement error was analysed using Dahlberg's formula and intraclass correlation coefficient (ICC). The occurrence of bias (systematic error) was assessed using paired t-tests.

Independent t-tests were used to compare the section areas at cervical levels 0,2 , and $4 \mathrm{~mm}$ between subjects from extraction and non-extraction groups, while paired t-tests were used to assess changes from $\mathrm{T} 1$ to $\mathrm{T} 2$ in groups. Correlational analysis and regression models were used to assess the relationship between the section areas at cervical levels 0,2 , and $4 \mathrm{~mm}$ and changes during treatment (from $\mathrm{T} 1$ to T2). All tests were interpreted at the 0.05 level of significance. The Excel 2013 (Microsoft, Redmond, WA, USA), SPSS (Statistical Package for the Social Sciences), Statistics 23 (IBM, Armonk, NY, USA), and MedCalc software (Mariakerke, Belgium) were used for statistical analysis. 
Table 1. Area of the section of the alveolar process measured pre-treatment in the whole sample $(n=75)$

\begin{tabular}{lccccccccccc}
\hline Level & Mean & SD & Minimum & Maximum & \multicolumn{7}{c}{ Percentiles } \\
\cline { 5 - 12 } & & & & & $\mathbf{5}$ & $\mathbf{1 0}$ & $\mathbf{2 5}$ & $\mathbf{5 0}$ & $\mathbf{7 5}$ & $\mathbf{9 0}$ & $\mathbf{9 5}$ \\
\hline 0 & 850.8 & 106.9 & 660.1 & 1223.8 & 682.6 & 714.2 & 773.0 & 840.7 & 918.3 & 1010.0 & 1023.4 \\
2 & 931.3 & 98.1 & 737.3 & 1325.4 & 786.5 & 814.7 & 863.9 & 920.3 & 981.5 & 1047.4 & 1100.1 \\
4 & 959.1 & 101.4 & 764.7 & 1368.5 & 817.0 & 833.8 & 881.1 & 953.5 & 1009.4 & 1067.1 & 1120.2 \\
\hline
\end{tabular}

Table 2. Changes during treatment in the whole sample and extraction and non-extraction groups

\begin{tabular}{lcccc}
\hline & $\begin{array}{c}\text { Whole sample } \\
(\mathbf{n}=\mathbf{7 5})\end{array}$ & $\begin{array}{c}\text { Extraction treatment } \\
\mathbf{( n = 3 6 )}\end{array}$ & $\begin{array}{c}\text { Non-extraction treatment } \\
(\mathbf{n}=39)\end{array}$ & $\begin{array}{c}\text { Differences between groups } \\
(\mathbf{p} \text { value and 95\% Cl's) }\end{array}$ \\
\hline Level 0 mm & & & & \\
Pretreatment (SD) & $850.8(106.9)$ & $865.7(95.7)$ & $836.9(115.7)$ & $0.246(-20.3 \ldots 77.9)$ \\
Posttreatment (SD) & $841(107.7)$ & $835.4(105.4)$ & $846.2(110.8)$ & $0.667(-60.7 \ldots 39.1)$ \\
Difference (SD) & $-9.8(45.6)$ & $-30.4(46.2)$ & $9.3(36.2)$ & $<0.001(-58.6 \ldots-20.6)$ \\
P value; 95\% Cl & $0.068(-0.7 \ldots 20.2)$ & $<0.001(14.7 \ldots 46)$ & $0.119(-21 \ldots 2.5)$ & \\
Level 2 mm & & & & \\
Pretreatment (SD) & $931.3(98.1)$ & $932.7(87.1)$ & $929.9(108.3)$ & $0.902(-42.6 \ldots 48.3)$ \\
Posttreatment (SD) & $922.8(96.6)$ & $909.5(91.9)$ & $935.1(100.4)$ & $0.255(-70 \ldots 18.8)$ \\
Difference (SD) & $-8.6(36.1)$ & $-23.2(37.5)$ & $5(29.2)$ & $<0.001(-43.6 \ldots-12.8)$ \\
P value; 95\% Cl & $0.046(0.2 \ldots 16.8)$ & $<0.001(10.6 \ldots 35.9)$ & $0.276(-14.6 \ldots 4.3)$ & \\
Level 4 mm & & & & \\
Pretreatment (SD) & $959.1(101.4)$ & $950.7(90.8)$ & $966.7(110.9)$ & $0.498(-62.9 \ldots 30.9)$ \\
Posttreatment (SD) & $952.3(99.7)$ & $938.3(94.4)$ & $965.1(103.9)$ & $0.248(-72.6 \ldots 19.1)$ \\
Difference (SD) & $-6.8(34.6)$ & $-12.4(38.3)$ & $-1.6(30.3)$ & $0.18(-26.6 \ldots 5.1)$ \\
P value; 95\% Cl & $0.093(-1.2 \ldots 14.7)$ & $0.061(-0.6 \ldots 25.3)$ & $0.736(-8.2 \ldots 11.5)$ & \\
\hline
\end{tabular}

$\mathrm{Cl}$ — confidence interval; $\mathrm{SD}$ - standard deviation

\section{RESULTS}

\section{Reliability of the method}

Measurement error ranged from 0.650 to 0.798 according to Dahlberg's formula. The ICCs ranged from 0.998 to 1 , implying almost perfect reliability. No systematic error was found with paired t-tests ( $p>0.05$ ). Intra-observer measurement variability ranged from 0.83 to 0.97 for the section area at levels 2 and 0 , respectively.

\section{Pre-treatment morphology}

Mean values, standard deviations, ranges, and percentile distributions of the pre-treatment area of the alveolus section surface measured at levels 0,2 , and 4 are presented in Table 1 . The areas were highly correlated with each other - correlation coefficients ranged from 0.797 (levels 0 and 4) to 0.942 (levels 0 and 2), and to 0.945 (levels 2 and 4). Table 2 demonstrates pre-treatment measurements in the whole sample and groups. The mean area of the alveolus section before treatment was smallest at level 0 and largest at level 4 . No differences were observed between extraction and non-extraction groups $(p=0.246,0.902$, and 0.498 for levels 0,2 , and 4 , respectively).

\section{Changes during treatment}

Changes during treatment are presented in Table 2. The area of the section decreased in the whole sample at all levels $(0,2$, and 4$)$. However, only the change registered at level 2 was statistically significant ( $p=0.046$; Table 2 ). In the extraction group, changes occurring during treatment were statistically significant for levels 0 and 2 ( $p<0.001$ and $p<0.001$, respectively), while those observed at level 4 were statistically non-significant $(p=0.061)$. Comparison of changes in extraction and non-extraction groups showed that those registered at levels 0 and 2 were statistically highly significant $(p<0.001)$, while changes measured at level 4 were statistically non-significant $(p=0.18)$.

\section{Relationship between pre-treatment morphology and the number of changes during treatment}

Regression models (Table 3 ) with treatment alternatives (extraction vs. non-extraction) and pre- 
Table 3. Regression models with the change of the area during treatment (measured at levels 0,2 , and 4 ) as dependent variable and area before treatment (measured at levels 0,2 , and 4 ) and treatment alternative (extraction vs. non-extraction) as independent variables

\begin{tabular}{cccccccccc}
\hline Level & $\begin{array}{c}\text { Independent } \\
\text { variables }\end{array}$ & $\begin{array}{c}\text { Unstanda- } \\
\text { ridsed B }\end{array}$ & SE & Standardised B & p value & $\begin{array}{c}\text { 95\% Cl lower } \\
\text { bound }\end{array}$ & $\begin{array}{c}\text { 95\% Cl upper } \\
\text { bound }\end{array}$ & $\begin{array}{c}\mathbf{p} \text { of the } \\
\text { model }\end{array}$ & $\begin{array}{c}\mathbf{R}^{2} \text { of the } \\
\text { model }\end{array}$ \\
\hline \multirow{2}{*}{0} & Area pre-treatment & -0.06 & 0.05 & -1.31 & 0.193 & -0.15 & 0.03 & $<0.001$ & 0.21 \\
\hline \multirow{2}{*}{2} & (1) vs. (2) & 37.91 & 9.58 & 3.96 & $<0.001$ & 18.81 & 57.01 & & \\
\hline \multirow{2}{*}{4} & Area pre-treatment & -0.08 & 0.04 & -2.04 & 0.045 & -0.16 & 0.00 & $<0.001$ & 0.2 \\
& Area pre-treatment & -0.08 & 0.04 & -2.05 & 0.044 & -0.16 & 0.00 & \multirow{2}{*}{0.053} & 0.08 \\
\hline
\end{tabular}

$\mathrm{Cl}$ — confidence interval; SE — standard error; (1) — extraction group; (2) — non-extraction group

treatment area of the alveolus (at levels 0,2 , and 4, respectively) as independent variables and the change in section surface of the alveolus during treatment (at levels 0, 2, and 4, respectively) as dependent variables showed that the pre-treatment area of the alveolus was a predictor of change during treatment only at level 2 (at level 4, the model was statistically non-significant). However, the effect of the pre-treatment area of the alveolus on dependent variables was limited - standardised B coefficient was -2.04 and $R^{2}$ of the model was 0.2 . Treatment alternatives affected changes in the section surface of the alveolus during treatment, measured at levels 0 and 2 - non-extraction treatment was associated with larger increases of $37.9 \mathrm{~mm}^{2}$ (level 0 ) and $28 \mathrm{~mm}^{2}$ (level 2) in comparison to extraction treatment. It should be noted that regression models explained approximately $20 \%$ of the variance in the sample.

\section{DISCUSSION}

One of the axioms in orthodontics holds that the bone follows the tooth during its movement through the alveolus. This is possible thanks to bone remodelling - a coupled process of bone resorption and apposition elicited by the mechanical force applied to the tooth by an orthodontic appliance $[9,22]$. The ratio between remodelling of the alveolar process and tooth movement is claimed to be $1: 1$. If this ratio is preserved, the root of the tooth is always supported by the alveolar bone and no bone loss occurs during orthodontic treatment [4]. However, this ratio is not achieved every time in all types of tooth movement, or in all areas of the alveolar process. For example, studies on the effects of tooth movement across the alveolus demonstrate that when the tooth apices were displaced into cortical bone, through penetration, the roots' surface became exposed. Reverse movement of the roots into the cancellous bone of the alveolus allowed for partial osteogenesis [19]. However, it was not sufficient to cover the root fully [20]. The lack of full regeneration of the cortical bone was observed even several years after treatment [5]. An unfavourable consequence of this phenomenon can be periodontal breakdown, particularly when inflammation of the periodontal tissues coincides.

Based on the results of this study, it is proposed that for patients in whom the surface area of the alveolus on pre-treatment digital models is measured at level 0 at less than $773 \mathrm{~mm}^{2}$, level 2 at less than $863.9 \mathrm{~mm}^{2}$, and level 4 at less than $881.1 \mathrm{~mm}^{2}$, the tooth movement across the alveolar process (i.e. proclination/retroclination of anterior teeth, expansion of posterior dental segment) should be planned with particular caution. In such patients, the area of the alveolus is significantly below average (it lies within the $25^{\text {th }}$ percentile) and one can reasonably assume that the possibility of bringing the root into the cortical bone, or even worse - penetrating it, is increased in comparison to patients with a thick alveolar process. On the other hand, for patients in whom the surface area of the alveolar process at level 0 measures more than $918.3 \mathrm{~mm}^{2}$, at level 2 more than $981.5 \mathrm{~mm}^{2}$, and at level 4 is more than $1009.4 \mathrm{~mm}^{2}$, the teeth can have less constraint from the cortical bone, hence, less caution may be required during planning and execution of orthodontic treatment.

Extraction of teeth is frequently associated with palatal movement of the remainder $[2,17]$. The results of this study confirm that extractions of maxillary first premolars followed by orthodontic space closure cause a decrease in the section surface area of the alveolus. The mean percentage reduction of the pre-treatment area was $<5 \%$. Nevertheless, caution is warranted in patients with a thin (as described above) alveolar 
process. In these patients, palatal movement of the remaining teeth could result in dangerous approximation of the root surfaces and the cortical bone with ensuing formation of bone dehiscence/fenestration [8].

The findings demonstrate that in a sample of orthodontic patients with typical malocclusion (Angle Class II Division 1 and Angle Class II Division 2 treated with and without the extraction of maxillary first premolars) the treatment alternatives of extraction vs. non-extraction are better predictors of changes in the surface section area of the alveolar process than its pre-treatment area. In other words, when a clinician plans a patient's treatment, he/she needs to carefully consider the possible consequences of extraction and non-extraction treatment alternatives for the alveolus, especially for patients with a thin alveolar morphotype.

While radiography represents a "gold standard" for the evaluation of changes in the alveolar region during orthodontic therapy, it is associated with exposure to potentially harmful ionising radiation. In the present study, the authors attempt to create a noninvasive and simple method for monitoring changes in the alveolar bone during orthodontic therapy. To our knowledge, there are no current studies focusing on the measurement of the entire alveolar process horizontally using a 3D scanner.

\section{Limitations of the study}

In this study, extraction and non-extraction groups comprise subjects with Angle Class II Division 1 and Angle Class II Division 2 malocclusion. Pre-treatment inclination of maxillary central incisors - proclined in Division 1 and retroclined in Division 2 malocclusion - could have affected measurement of the pre-treatment area of section surface area of the alveolus. In order to prevent this, the ratio of patients was balanced with Angle Class II Division 1 and Angle Class II Division 2 malocclusion in the extraction and non-extraction groups.

Only the post-adolescent subjects in this investigation were evaluated. Therefore, the results can be generalised only for subjects at a similar developmental stage.

The assessed changes of the alveolar process were based on the scans of its external surface. Thus, any measurement of the section surface area of the alveolus is a combination of the measurement through soft tissues and the underlying alveolar bone.

\section{CONCLUSIONS}

Within the limitations of the study the following conclusions can be drawn: (a) Digital model analysis of the dental arch should be used in planning orthodontic treatment; (b) Patients for whom the surface area of the alveolar process is measured on pre-treatment digital models at $<773 \mathrm{~mm}^{2},<863.9 \mathrm{~mm}^{2}$, and $<881.1 \mathrm{~mm}^{2}$ at levels 0,2 , and 4, respectively, should be described as having a "thin" alveolus; (c) Extraction treatment, which is associated with a decrease in the alveolus area, should be exercised with caution in subjects with a thin alveolus.

\section{Acknowledgements}

This work was in part supported by LO1304 from Ministry of Education, Youth and Sports, Czech Republic. We thank Vladimir Divoky, PhD (Faculty of Medicine and Dentistry, Palacký University, Olomouc) for his help with translation of the original research findings and for his suggestions towards manuscript finalisation, prof. Milan Kaminek, MD (Faculty of Medicine and Dentistry, Palacký University, Olomouc) for helpful discussions, and Hana Böhmova, MD (Faculty Hospital, Pilsen) for her technical assistance.

\section{REFERENCES}

1. Aarts BE, Convens J, Bronkhorst EM, et al. Cessation of facial growth in subjects with short, average, and long facial types - Implications for the timing of implant placement. J Craniomaxillofac Surg. 2015; 43(10): 2106-2111, doi: 10.1016/j.jcms.2015.10.013, indexed in Pubmed: 26548528.

2. Ahn HW, Moon SC, Baek SH. Morphometric evaluation of changes in the alveolar bone and roots of the maxillary anterior teeth before and after en masse retraction using cone-beam computed tomography. Angle Orthod. 2013; 83(2): 212-221, doi: 10.2319/041812-325.1, indexed in Pubmed: 23066654.

3. Bailey LT, Esmailnejad A, Almeida MA. Stability of the palatal rugae as landmarks for analysis of dental casts in extraction and nonextraction cases. Angle Orthod. 1996; 66(1): 73-78, doi: 10.1043/0003-3219(1996)066<0073:SOTPR A>2.3.CO;2, indexed in Pubmed: 8678349.

4. Baloul S. Osteoclastogenesis and osteogenesis during tooth movement. Tooth Movement. 2016; 18: 75-79, doi: $10.1159 / 000351901$.

5. Duterloo HS. The impact of orthodontic treatment procedures on the remodelling of alveolar bone. Orthodontische Studieweek. Ned Ver Orthod Studie. 1975: 5-21.

6. Grauer D, Cevidanes LH, Tyndall D, et al. Registration of orthodontic digital models. Craniofac Growth Ser. 2011; 48: 377-391, indexed in Pubmed: 26549917.

7. Güleç $A$, Kaçıra BK, Kütahya $H$, et al. Morphometric analysis of the lumbar vertebrae in the Turkish population using 
three-dimensional computed tomography: correlation with sex, age, and height. Folia Morphol. 2017; 76(3): 433-439, doi: 10.5603/FM.a2017.0005, indexed in Pubmed: 28150271.

8. Handelman CS. The anterior alveolus: its importance in limiting orthodontic treatment and its influence on the occurrence of iatrogenic sequelae. Angle Orthod. 1996; 66(2): 95-109; discussion 109, doi: 10.1043/0003-3219(1996)066<0095:TAAIII>2.3. CO;2, indexed in Pubmed: 8712499.

9. Krishnan V, Davidovitch Z. On a path to unfolding the biological mechanisms of orthodontic tooth movement. J Dent Res. 2009; 88(7): 597-608, doi: 10.1177/0022034509338914, indexed in Pubmed: 19641146.

10. Kuijpers MAR, Chiu YT, Nada RM, et al. Three-dimensional imaging methods for quantitative analysis of facial soft tissues and skeletal morphology in patients with orofacial clefts: a systematic review. PLoS One. 2014; 9(4): e93442, doi: 10.1371/journal.pone.0093442, indexed in Pubmed: 24710215.

11. Marinković S, Milić I, Djorić I, et al. Morphometric multislice computed tomography examination of the craniovertebral junction in neck flexion and extension. Folia Morphol. 2017; 76(1): 100-109, doi: 10.5603/FM.a2016.0037, indexed in Pubmed: 27830891.

12. Nouri M, Abdi AH, Farzan A, et al. Measurement of the buccolingual inclination of teeth: manual technique vs 3-dimensional software. Am J Orthod Dentofacial Orthop. 2014; 146(4): 522-529, doi: 10.1016/j.ajodo.2014.06.018, indexed in Pubmed: 25263155.

13. Pachêco-Pereira C, De Luca Canto G, Major PW, et al. Variation of orthodontic treatment decision-making based on dental model type: A systematic review. Angle Orthod. 2015; 85(3): 501-509, doi: 10.2319/051214-343.1, indexed in Pubmed: 25098186.

14. Rischen RJ, Breuning $\mathrm{KH}$, Bronkhorst EM, et al. Records needed for orthodontic diagnosis and treatment planning: a systematic review. PLoS One. 2013; 8(11): e74186, doi: 10.1371/journal.pone.0074186, indexed in Pubmed: 24265669.

15. Sadek MM, Sabet NE, Hassan IT, et al. Alveolar bone mapping in subjects with different vertical facial dimensions. Eur J Orthod. 2015; 37(2): 194-201, doi: 10.1093/ejo/ cju034, indexed in Pubmed: 25114124.

16. Salti L, Holtfreter B, Pink C, et al. Estimating effects of craniofacial morphology on gingival recession and clinical attachment loss. J Clin Periodontol. 2017; 44(4): 363-371, doi: 10.1111/jcpe.12661, indexed in Pubmed: 27930822.

17. Sarikaya S, Haydar B, Ciğer S, et al. Changes in alveolar bone thickness due to retraction of anterior teeth. Am J Orthod Dentofacial Orthop. 2002; 122(1): 15-26, indexed in Pubmed: 12142888.

18. Swasty D, Lee J, Huang JC, et al. Cross-sectional human mandibular morphology as assessed in vivo by conebeam computed tomography in patients with different vertical facial dimensions. Am J Orthod Dentofacial Orthop. 2011; 139(4 Suppl): e377-e389, doi: 10.1016/j. ajodo.2009.10.039, indexed in Pubmed: 21435546.

19. Thilander B, Nyman S, Karring T, et al. Bone regeneration in alveolar bone dehiscences related to orthodontic tooth movements. Eur J Orthod. 1983; 5(2): 105-114, indexed in Pubmed: 6574916.

20. Wainwright WM. Faciolingual tooth movement: its influence on the root and cortical plate. Am J Orthod. 1973; 64(3): 278-302, indexed in Pubmed: 4199008.

21. Wennström JL. Mucogingival considerations in orthodontic treatment. Semin Orthod. 1996; 2(1): 46-54, indexed in Pubmed: 9161283.

22. Wise GE, King GJ. Mechanisms of tooth eruption and orthodontic tooth movement. J Dent Res. 2008; 87(5): 414-434, doi: 10.1177/154405910808700509, indexed in Pubmed: 18434571.

23. Zhang S, Wang X, Ren X, et al. Applications of digital technology for the morphological study of C3-C7 vertebral arch pedicle in children. Folia Morphol (Warsz). 2017; 76(3): 426-432, doi: 10.5603/FM.a2017.0003, indexed in Pubmed: 28150269. 